\title{
How to use abdominal X-rays in preterm infants suspected of developing necrotising enterocolitis
}

\author{
Roopali Soni, ${ }^{1}$ Anastasia Katana, ${ }^{1}$ Joe I Curry, ${ }^{2}$ Paul D Humphries, ${ }^{3}$ \\ Angela Huertas-Ceballos ${ }^{1}$
}

\begin{abstract}
${ }^{1}$ Department of Neonatology, University College London Hospital, London, UK ${ }^{2}$ Department of Paediatric Surgery, Great Ormond Street Hospital, London, UK ${ }^{3}$ Department of Radiology, Great Ormond Street Hospital, London, UK
\end{abstract}

\section{Correspondence to}

Dr Angela Huertas-Ceballos, Department of Neonatology, University College London Hospital, London WC1E 6DB, UK; a.huertas-ceballos@nhs.net

Accepted 16 December 2018 Published Online First 18 February 2019

\section{A Check for updates}

(c) Author(s) (or their employer(s)) 2020. No commercial re-use. See rights and permissions. Published by BMJ.

To cite: Soni $R$, Katana $A$,

Curry Jl, et al. Arch Dis

Child Educ Pract Ed

2020;105:50-57.

\section{ABSTRACT}

Necrotising enterocolitis (NEC) in preterm babies is a serious gastrointestinal emergency with potentially devastating consequences. Prompt and accurate diagnosis continues to be a challenge for health professionals. Early identification of clinical warning signs is extremely important, but the diagnosis relies heavily on the interpretation of abdominal radiographs. Postgraduate training of paediatricians and neonatologists in neonatal abdominal radiography is scarce, and there is variability of radiological input to neonatal services. Lack of a standardised approach and descriptive terminology for interpretation may result in inadequate communication between clinical and surgical teams, inaccurate diagnosis, inappropriate treatment, and unnecessary cessation of feeds and transfers to surgical units. This paper offers a guide designed for the doctor who on a busy night shift needs to interpret an abdominal radiograph and decide on a differential diagnosis of NEC in a preterm baby. It helps to provide structure and standardisation to interpretation of radiological signs using a comprehensive but simple method to support the clinical diagnosis. Our aim is to enhance the correct diagnosis of NEC.

\section{INTRODUCTION}

Necrotising enterocolitis (NEC) is a serious life-threatening gastrointestinal disease in the newborn, affecting 1\%-5\% of neonatal intensive care unit admissions and up to $10 \%$ of neonates under $1500 \mathrm{~g} .{ }^{1}$ The incidence and severity of NEC rise in inverse relationship to gestational maturity. The overall mortality rate in NEC is between $20 \%$ and $40 \%$, approaching $50 \%$ in extremely low birthweight infants, who require surgical treatment of NEC. ${ }^{2}$ Approximately $25 \%$ of NEC survivors suffer from serious comorbidities including short gut syndrome and neurodevelopment impairment. ${ }^{3-5}$

The current diagnosis of NEC is based on the modified Bell's criteria ${ }^{67}$ (table 1), which include clinical, pathophysiological and radiological signs, and classify the severity of the disease in stages from I (suspicion of NEC) to IIIB (perforated NEC). The system, based on the surgical

Table 1 Modified Bell's staging criteria (Kliegman and Walsh ${ }^{7}$ )

\begin{tabular}{|c|c|c|c|}
\hline Modified Bell's staging & Clinical findings & Radiographic findings & Gastrointestinal findings \\
\hline Stage I & $\begin{array}{l}\text { Apnoea, bradycardia and } \\
\text { temperature instability. }\end{array}$ & $\begin{array}{l}\text { Normal gas pattern or mild } \\
\text { ileus. }\end{array}$ & $\begin{array}{l}\text { Mild abdominal distension, } \\
\text { stool occult blood, gastric } \\
\text { residuals. }\end{array}$ \\
\hline Stage IIA & $\begin{array}{l}\text { Apnoea, bradycardia and } \\
\text { temperature instability. }\end{array}$ & $\begin{array}{l}\text { lleus with dilated } \\
\text { bowel loops and focal } \\
\text { pneumatosis. }\end{array}$ & $\begin{array}{l}\text { Moderate abdominal } \\
\text { distension, haematochezia, } \\
\text { absent bowel sounds. }\end{array}$ \\
\hline Stage IIB & $\begin{array}{l}\text { Metabolic acidosis and } \\
\text { thrombocytopaenia. }\end{array}$ & $\begin{array}{l}\text { Widespread pneumatosis, } \\
\text { portal venous gas, ascites. }\end{array}$ & $\begin{array}{l}\text { Abdominal tenderness and } \\
\text { oedema. }\end{array}$ \\
\hline Stage IIIA & $\begin{array}{l}\text { Mixed acidosis, } \\
\text { coagulopathy, hypotension, } \\
\text { oliguria. }\end{array}$ & $\begin{array}{l}\text { Moderate to severely } \\
\text { dilated bowel loops, } \\
\text { ascites, no free air. }\end{array}$ & $\begin{array}{l}\text { Abdominal wall oedema, } \\
\text { erythema and induration. }\end{array}$ \\
\hline Stage IIIB & $\begin{array}{l}\text { Shock, worsening vital } \\
\text { signs and laboratory } \\
\text { values. }\end{array}$ & Pneumoperitoneum. & Bowel perforation. \\
\hline
\end{tabular}




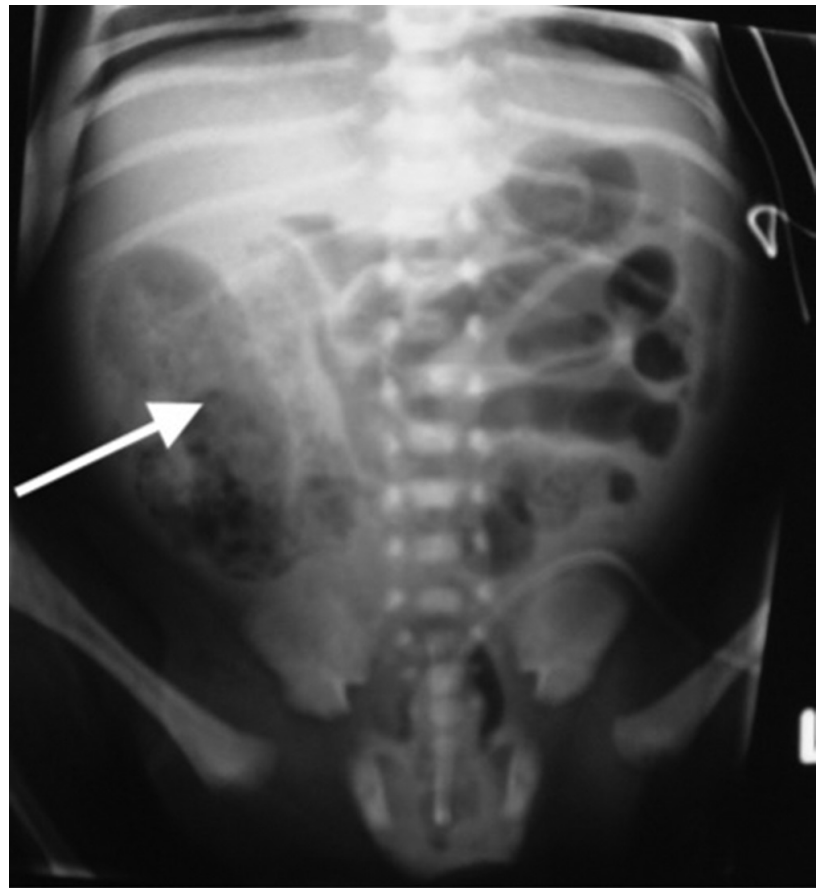

Figure 1 Soap bubble appearance (arrow) persisting at 48 hours into treatment for necrotising enterocolitis. Found to be necrotic ascending colon at laparotomy.

description of 48 cases published in 1950, is based on categorical criteria for X-ray appearances, which are not always straightforward. The current surviving population of extreme premature infants often have degrees of intestinal dysmotility that produce abnormal X-ray changes that are not in themselves secondary to NEC but are prone to misinterpretation.

Abdominal X-rays (AXRs) are heavily relied on to help establish a diagnosis of NEC. Interpretation of plain abdominal radiographs in premature infants is usually challenging, and as a consequence feeds may be suspended for several days in many of these babies because of a concern of NEC, impairing both nutritional and neurodevelopment outcomes. ${ }^{4}$ Correct identification of specific signs on abdominal radiographs and consistency in terminology are therefore paramount.

In this article we offer guidance for interpreting abdominal radiographs in preterm babies where NEC is a differential diagnosis.

\section{PATHOLOGICAL CORRELATION WITH RADIOGRAPHY}

In NEC, bacteria penetrate the mucosal defence, and their by-products of metabolism lead to the formation of intramural gas (figures 1-3). As NEC progresses, the inflammatory cascade leads to transmural involvement. Eventually, ischaemic changes take place due to compromise of the microvasculature. Necrosis of the non-perfused bowel wall finally takes place and may be so severe that sloughing of the bowel wall occurs, eventually leading to perforation (figures 4-6). ${ }^{1}$

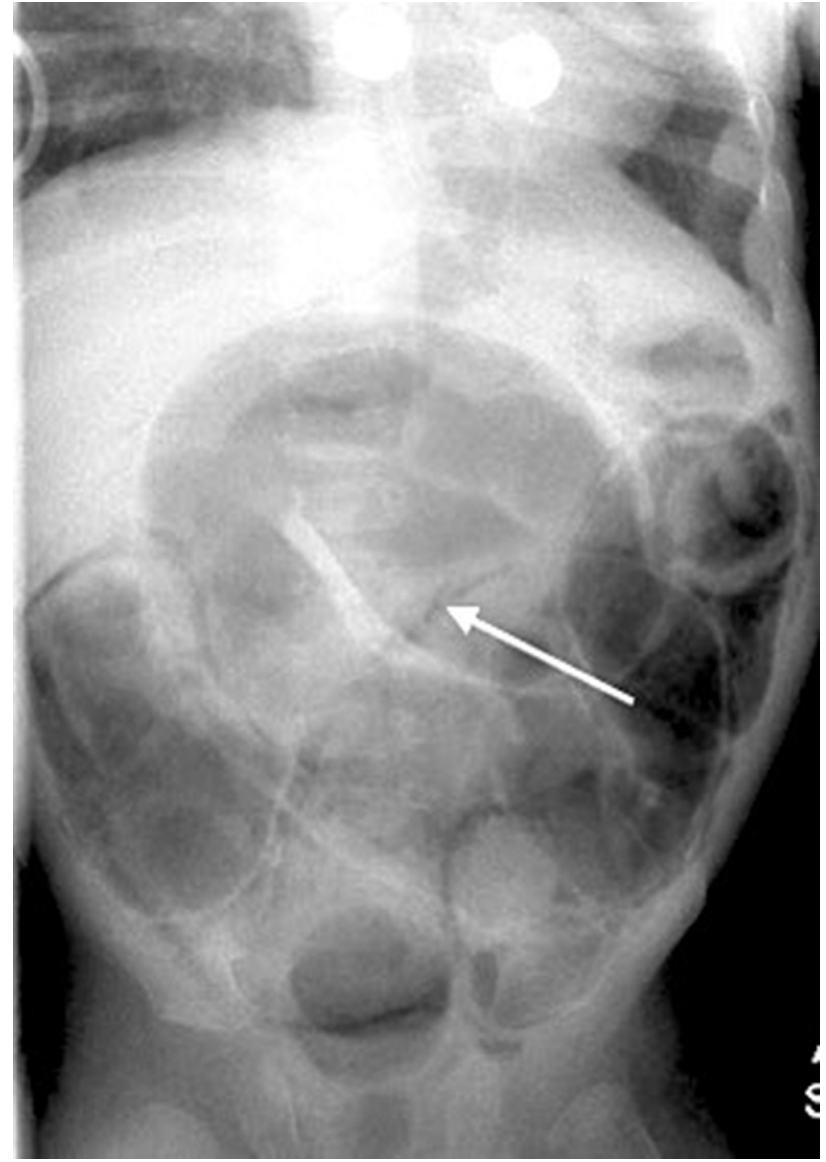

Figure 2 Widespread pneumatosis seen as crescents (arrow).

There is strong evidence of a multifactorial aetiology. Predisposing factors include genetic susceptibility, intestinal immaturity, imbalance in microvascular tone, abnormal microbial colonisation of the intestines and a highly immunoreactive intestinal mucosa. ${ }^{8}$

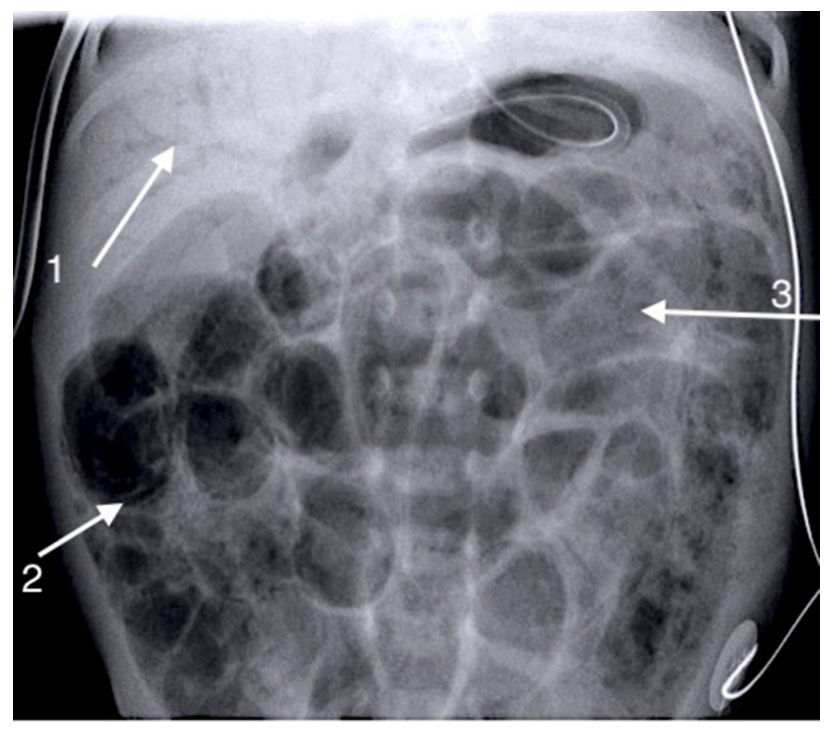

Figure 3 Arrow 1 shows the branching tree of the portal venous gas. Arrow 2 shows the crescents of pneumatosis. Arrow 3 shows widespread soap bubbling. 


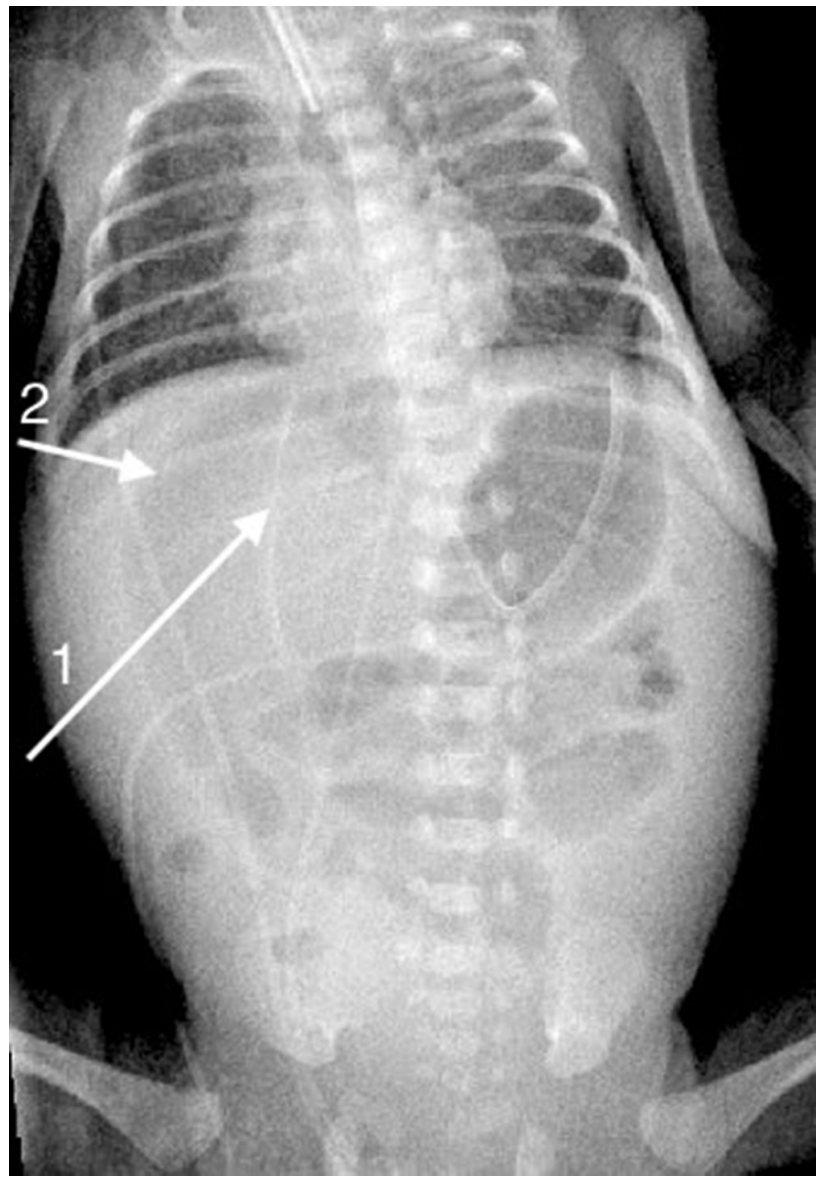

Figure 4 The 'football sign' is demonstrated with gas on either side of the falciform ligament (arrow 1). Arrow 2 shows the lucency over the liver, indicating pneumoperitoneum.

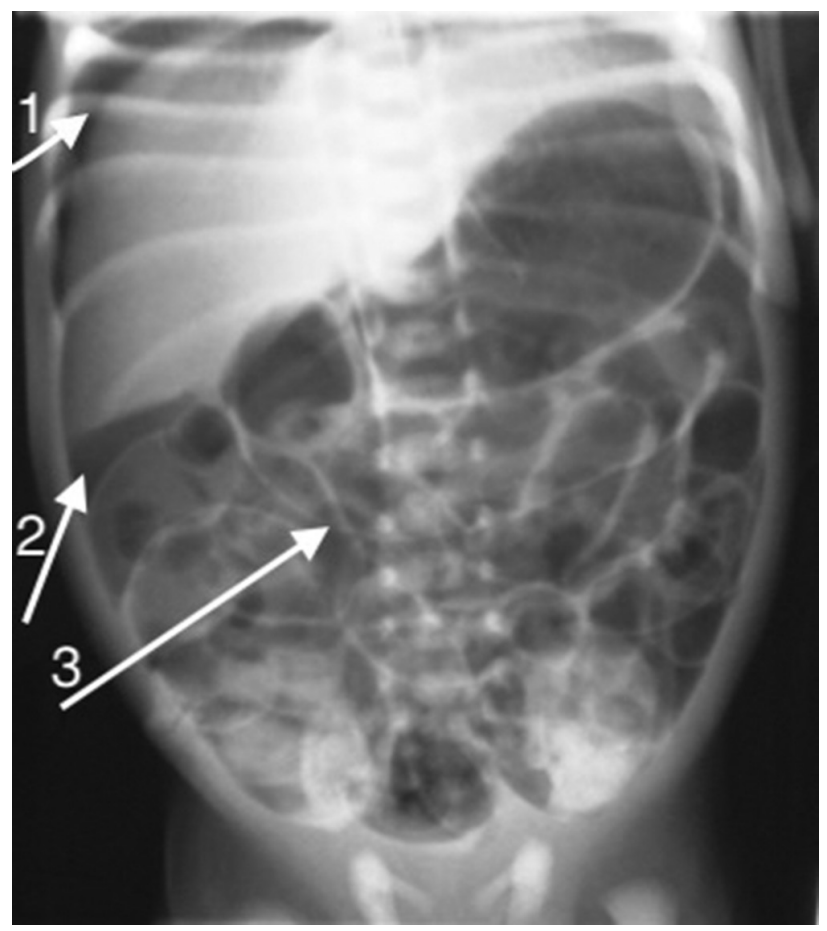

Figure 5 Arrow 1 shows gas under the diaphragm. Arrow 2 shows a 'triangle' under the liver. Arrow 3 points at the 'Rigler sign'.

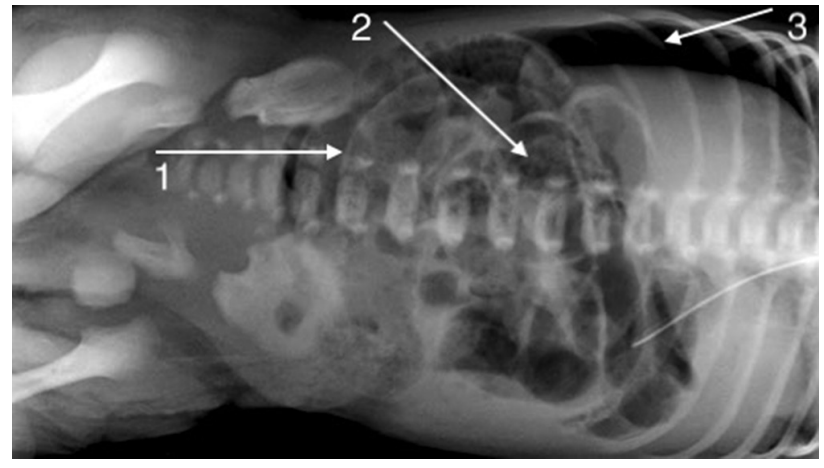

Figure $6 \quad$ X-ray taken lying on the left side. Arrow 1 shows the 'Rigler sign'. Arrow 2 shows widespread soap bubbling. Arrow 3 shows the solid liver clearly outlined by free intraperitoneal gas when the baby is placed right side up.

Many of the radiographic signs of NEC are dependent on the phenomenon of gas-forming organisms intruding on the tissue planes in the gut and thereby revealing their pathological presence. Pneumatosis intestinalis (intramural gas) in a neonate is considered a virtually pathognomonic radiographic sign of NEC. The bubbly appearance is due to submucosal gas 'blebs', whereas curvilinear lucencies in the bowel wall are due to gas in a subserosal location. The reported incidence of pneumatosis in cases of NEC varies from $19 \%$ to $98 \% .{ }^{19}$ However, in clinical practice, disagreement often occurs over signs on $\mathrm{X}$-rays when it is unclear if findings are suggestive of pneumatosis or not.

The other virtually pathognomonic sign is portal venous gas (PVG). It is not as early a sign as pneumatosis and may appear and disappear rapidly, and may easily be missed. ${ }^{19}$ PVG (portal venous gas) may also be seen when a UVC is in situ, where there is no prior suspicion of NEC.

Pneumoperitoneum is a frequent indication for surgical intervention, but is complicated by the fact that only in between one half and three quarters of patients with perforation is free air detectable even on lateral film. .Physiological deterioration despite maximal medical therapy remains a relative indication for operation. ${ }^{2}$

\section{RADIOGRAPHIC TECHNIQUE}

A supine anteroposterior film is recommended when NEC is suspected. Further AXRs (6-12 hourly), dependent on the clinical state of the infant, are recommended if there are signs of pneumatosis on the initial radiograph.

A lateral decubitus radiograph (right side up) may be useful when perforation is suspected but not clear on plain anteroposterior X-ray. A lateral shoot-through radiograph, with the baby supine rather than right side up, may be useful in case of an unstable infant where it would be beneficial to reduce or avoid movement. 
Box 1 Proposed guide: how to read abdominal $X$-rays where there is a suspicion of necrotising enterocolitis (NEC)

\section{A. General information.}

$\checkmark$ Identify.

- The patient.

- Date of the film.

- Time of the film.

- Check the adequacy of the film.

- Ensure that it is a straight film and appropriately centred.

- Make sure it includes all of the abdomen, tops of the diaphragms and the lung bases.

- Check the X-ray penetration.

- Note the presence of artefacts such as gel mattresses, monitoring equipment and so on.

- Assess the degree of rotation.

- Assess the orientation of the visible anterior ribs and the pelvic appearances to determine if there is significant rotation.

- Equipment.

- Identify the site and location of equipment such as gastric tubes, drains, urine catheters, long lines and umbilical catheters.

$\checkmark$ Chest.

- If visible, assess the chest for signs of pathology.

- Air.

- Stomach bubble on the left, unless suspected rotational abnormality.

- Bowel gas pattern.

- Normal: a reasonable amount of equal calibre gut throughout the abdomen*.

- Abnormal: any alteration to calibre, continuity, asymmetry or paucity.

Soft tissue.

- Liver should be located on the right upper quadrant and should cross the midline.

- Kidneys may be visible as soft tissue shadows.

- Bladder may be visible as a soft tissue density projected over the central pelvis, possibly rising into the abdomen.

- Bone.

- Spine: look for abnormal vertebrae and asymmetries.

- Sacrum: look for sacral anomalies.

- Ribs: number and shape.

- Abnormal shadows.

- Calcifications: are usually radio-opaque shadows.

- Mass.

- Areas of different opacities within a shadow.

- Mass effect when bowel is pushed all to one side.

\section{B. Signs of NEC.}

- Crescents (figures 2, 3 and 7): curvilinear appearance of intramural gas - pathognomonic of NEC. Crescents are more commonly seen in the right lower quadrant (NEC commonly affects the terminal ileum and the ascending colon). However, they may involve any part of the gastrointestinal tract including the stomach.

- Soap bubbles (figures 1, 3 and 6): symmetrical, small, in linear patterns-soap bubbles are not always specific of

\section{Box 1 Continued}

NEC, and therefore clinical correlation and presence of other radiological signs are required for a presumptive diagnosis of NEC. It is generally accepted that the bubbly appearance is due to submucosal gas 'blebs', whereas curvilinear lucencies in the bowel wall are due to gas in a subserosal location.

- The bubbly morphology may be confused with stool in a normal colon. This is more commonly seen in older infants and children. A follow-up film 6 hours later is recommended to observe changes in position in the case of stool. The bubble pattern in stools is more regular and uniform and with very small bubbles. Stools are irregular, and different textures can be appreciated.

- Gas in the portal venous system (figure 3): seen as a shadow on the liver area, like the branching of a tree upwards. This is typically appreciated in the periphery of the liver, compared with biliary air, which is centrally located. Care should be taken not to mistake bronchial shadows from the inferior lobe of the right lung for portal venous gas; these would appear as branches of a tree pointing downwards. Portal venous gas is seen easily in USS (Ultra sound scan).

\section{Signs of perforation (figures 4, 5, 6 and 8). ${ }^{16}$}

- Rigler sign: is a sharp demarcation of the bowel wall (almost like a line drawn with a pencil), usually one of the first signs of perforation and implies that there is air on both sides of the bowel wall.

- Football sign: a large ovoid or circular lucency over the liver or in the central part of the abdomen due to a large amount of free intraperitoneal air. Sometimes, but not always, the falciform ligament is outlined due to the air being present on either side of it. This is seen as a faint linear opacity situated longitudinally within the right upper abdomen, representing the seams or laces of an American football. ${ }^{17}$

- Air under the diaphragm: can be seen in an anteroposterior or lateral view. Lateral view, ask for right side up so that the solid liver is clearly outlined by free intraperitoneal gas.

- Triangle sign: when free air becomes trapped in between loops of bowel, it can give the appearance of triangular shapes. This is also commonly seen under the liver.

- Lucency over solid abdominal organs: lucency over a solid organ may be indicative of pneumoperitoneum. This may be of variable sizes and may not look like a football.

- Gas in patent processus vaginalis (PPV): gas in the PPV can be seen with perforation and may reach down to the scrotum in boys.

\section{Other important signs.}

- Fixed loop: the presence of a non-moving, persistently dilated loop has been reported as a hallmark of impending perforation. ${ }^{1819}$ It is a radiographic sign that should be interpreted with caution as such loops can normalise with medical treatment.

- Persistent pneumatosis (figure 1): pneumatosis visible on $X$-rays beyond 24 hours of institution of therapy may be an indication of severe ischaemia. 


\section{Box 1 Continued}

- Bowel dilatation: bowel dilatation is considered normal in premature babies on invasive and non-invasive ventilation. The normal calibre of the bowel loop should be equal to the measurement of either the width of $L 5$ or the distance between the top of $\mathrm{L} 1$ to the bottom of $\mathrm{L} 2$.

- Separation of the bowel loops (figure 9): an increase in peritoneal fluid between the loops. Commonly seen in generalised or systemic sepsis and not in itself an indication of primary gut pathology. It is often mistaken as 'thickened bowel wall'.

- Dilated bowel loops in the centre of the abdomen (described by some surgeons as the 'flowerpot' sign): implies that there is significant ascites and correlates with a sick baby.

- Dilated loops surrounding an area of the abdomen that looks radio-opaque ('inverse flower pot' sign): implies a mass occupying the central abdominal space.

\section{E. Some frequently used terminology.}

- Thickened bowel wall: often misinterpreted as oedematous bowel wall. Pathology specimens show that bowel loops affected by NEC are not oedematous but in fact separated from adjacent loops by intraperitoneal fluid.

- Mottling: a term commonly used in neonatal care but not clear or specific; preferred terminology is 'bubbly' or 'soap bubbles'.

It is useful to remember that when a sick neonate presents with abdominal distension, the 'abdominal' symptoms may also be secondary to non-abdominal pathology such as chest infection. Therefore, review the lung visible on the abdominal film.

*It is accepted that one cannot distinguish small and large intestines on a plain X-ray in an infant, as the normal haustral pattern of the colon is not seen. The position of the gut in the abdominal radiograph is not an indication of which part of the intestine it is.

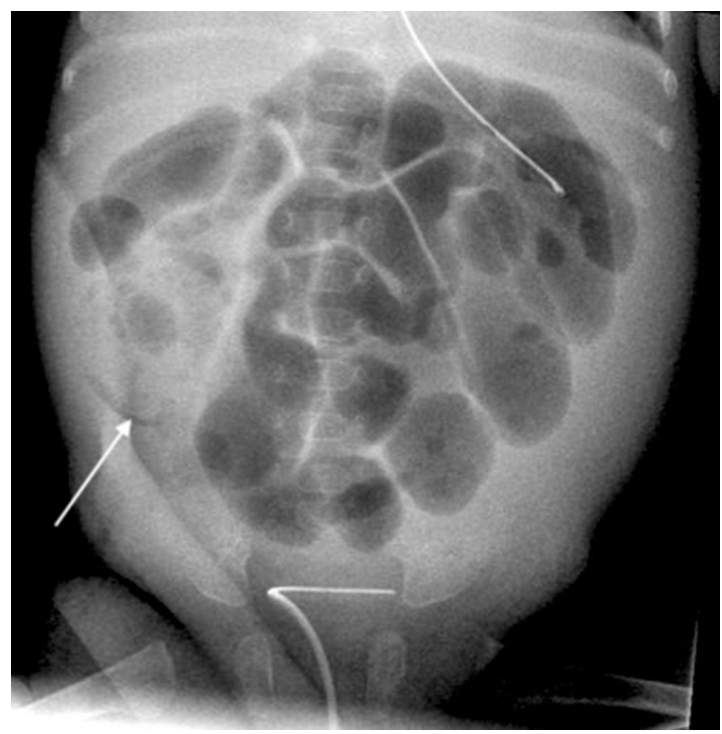

Figure 7 Crescent from pneumatosis.

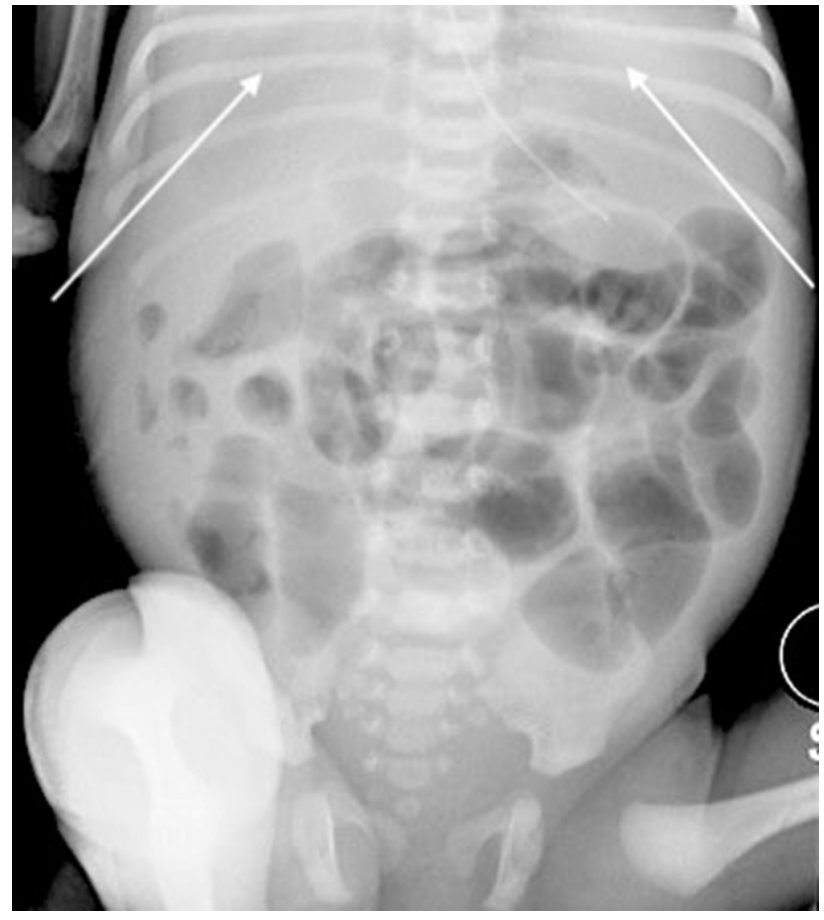

Figure 8 Both arrows show the very subtle difference in radiolucency indicating large gas bubble in front of the liver from perforation.

It is important to develop a methodical approach (box 1) to reading AXRs as it ensures that important structures are not ignored and findings that require immediate attention are recognised.

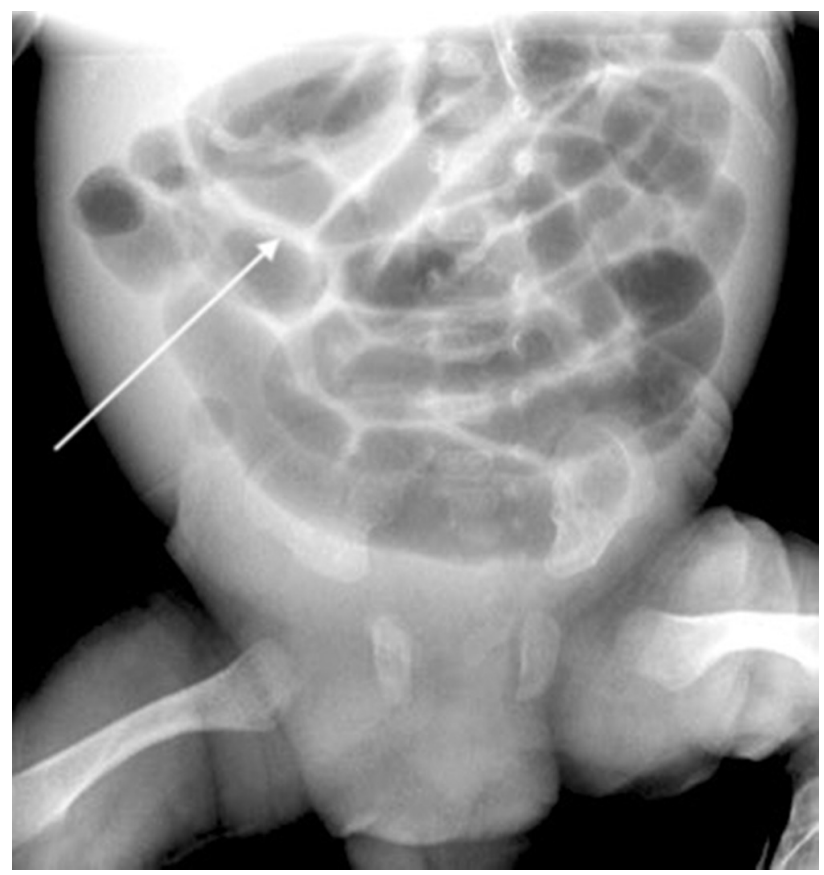

Figure 9 Infant with sepsis (not necrotising enterocolitis). The arrow point to the 'separation of bowel loops' by an increase in intraperitoneal fluid. 


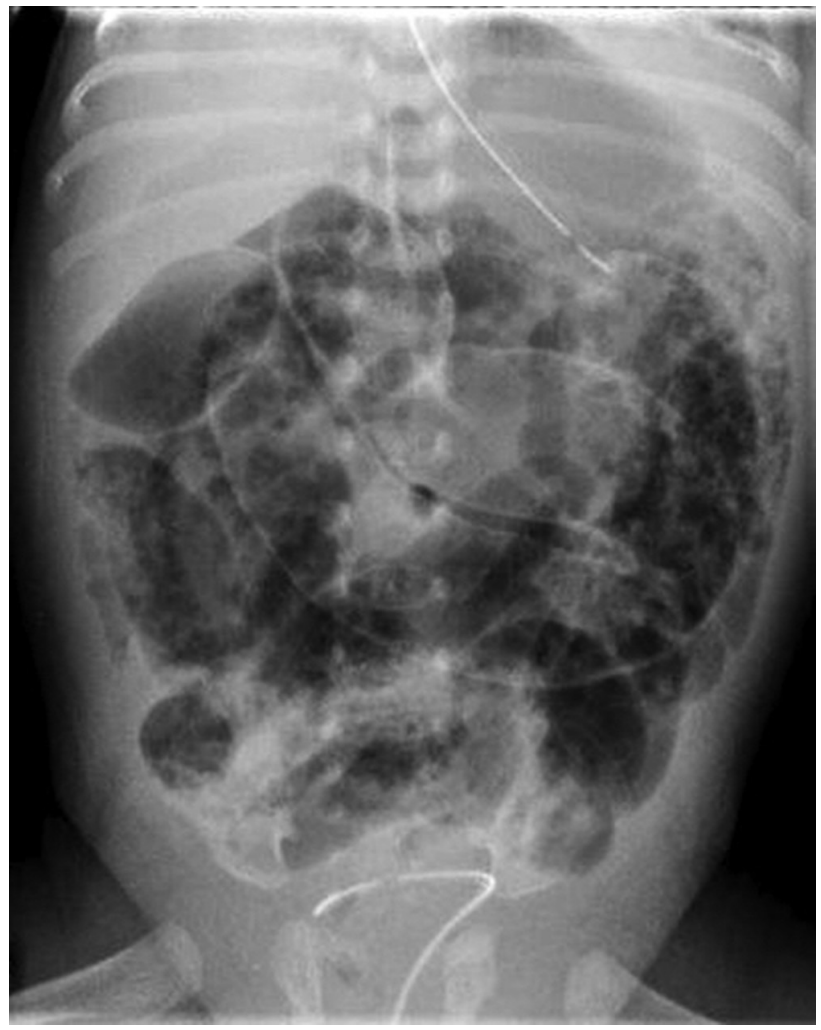

Figure 10 Multiple choice questions 4 and 5: abdominal X-ray of the infant.

\section{CLINICAL QUESTIONS}

In a preterm baby with non-specific symptoms, does a normal AXR rule out NEC?

NEC is a multisystem disease and the diagnosis relies on a full assessment of the infant with analysis of clinical and laboratory markers and radiology. The main problem with radiographic signs of NEC is that they might have a high positive predictive value (the highest values for pneumoperitoneum) but a very low sensitivity (less than 50\%). The X-ray should always be interpreted in conjunction with the clinical picture.

Tam et al ${ }^{10}$ reviewed the medical records of 80 infants undergoing exploratory laparotomy for presumed advanced NEC or focal intestinal perforation (FIP). Radiographic criteria were evaluated as predictors of NEC or perforation. They demonstrated high specificity but low sensitivity for radiographic signs traditionally associated with NEC and FIP (table 2).

In a baby with NEC, is resolution of pneumatosis and PVG on AXR a good sign?

In most patients the resolution of pneumatosis indicates clinical improvement, although in some babies the disappearance of pneumatosis coincides with clinical worsening. The amount of intramural gas present does not always relate to the clinical severity of NEC. ${ }^{19}$

PVG in NEC probably occurs in between 10\% and $30 \%$ of patients. It is commonly associated with more severe disease and a higher mortality than babies without PVG. However, as with pneumatosis, clinical improvement does not always accompany the disappearance of PVG on radiographs. ${ }^{19}$

In a preterm baby, should the presence of dilated bowel loops on AXR raise a suspicion of NEC?

Diffuse gaseous distension of the intestine is frequently suspected of indicating early or developing NEC. However, many premature babies who do not have NEC also have distended abdomen, particularly in the first 2 weeks of life. Low birthweight infants may frequently suffer from gaseous distension, bile-stained aspirates and intolerance to feeds in the absence of any detectable anatomical abnormality. Babies on nasal CPAP (continuous positive airway pressure) also often have distended abdomen. ${ }^{9}$

\section{Is 'bowel wall thickening' a reliable sign on AXR in a baby} with suspected NEC?

'Separation' of gas-filled bowel loops on radiography is often misinterpreted as being secondary to bowel wall thickening. In reality it is the increase in intraperitoneal fluid that creates this separation between the loops. It is a non-specific radiographic sign and should be interpreted with caution. It can be seen

\begin{tabular}{|c|c|c|c|c|c|c|}
\hline Radiological finding & Group & Sensitivity (\%) & Specificity (\%) & Positive predicative value (\%) & Negative predictive value (\%) & Prevalence $(\%)$ \\
\hline \multirow{3}{*}{ Pneumatosis } & All & $44(n=27)$ & $100(n=19)$ & 100 & 36 & 34 \\
\hline & EX LBW & $35(n=12)$ & $100(n=15)$ & 100 & 41 & 24 \\
\hline & LBW & $56(n=15)$ & $100(n=4)$ & 100 & 25 & 48 \\
\hline \multirow[t]{3}{*}{ Portal venous gas } & All & $13(n=8)$ & $100(n=19)$ & 100 & 26 & 10 \\
\hline & EX LBW & $12(n=4)$ & $100(n=15)$ & 100 & 50 & 8.1 \\
\hline & LBW & $15(n=4)$ & $100(n=4)$ & 100 & 15 & 13 \\
\hline \multirow[t]{3}{*}{ Pneumoperitoneum } & All & $52(n=23)$ & $92(n=33)$ & 88 & 61 & 29 \\
\hline & EX LBW & $47(n=15)$ & $100(n=17)$ & 100 & 50 & 31 \\
\hline & LBW & $67(n=8)$ & $84(n=16)$ & 73 & 80 & 26 \\
\hline \multirow[t]{3}{*}{ Gasless abdomen } & All & $32(n=14)$ & $92(n=33)$ & 82 & 52 & 18 \\
\hline & EX LBW & $41(n=13)$ & $82(n=14)$ & 81 & 42 & 27 \\
\hline & LBW & $8.3(n=1)$ & $100(n=19)$ & 100 & 63 & 28 \\
\hline
\end{tabular}

EX LBW, extremely low birth weight; LBW, low birth weight. 
in systemic sepsis and when the infant is generally oedematous. True bowel wall thickening is difficult to determine from plain abdominal radiographs and in fact is rarely seen in NEC. Abdominal ultrasound is a better modality to assess bowel wall thickness. ${ }^{11}$

\section{Are there any other diagnostic modalities that may be useful in the management of a baby where NEC is a possible diagnosis?}

Abdominal ultrasonography (AUS) has been reported as a valuable tool for depicting the presence of focal fluid collections and free gas, particularly in those patients who might have already perforated but have no evidence of this on AXR. Furthermore, it is useful in studying bowel wall thickness, perfusion (colour and power Doppler imaging) and peristalsis, all of which cannot be assessed on X-ray. PVG is also easily seen on AUS. Relative limitations include difficulty in imaging when there is a large amount of bowel gas and the potential to cause abdominal discomfort in a labile patient. ${ }^{1112}$

\section{LIMITATIONS}

The recognised AXR changes in NEC have a good positive predictive value but low sensitivity. Frequently in clinical practice, however, the changes may be subtle and difficult to interpret. Other more non-specific signs are frequently misinterpreted as indicating NEC, and the clinical experience of the treating physician can be paramount in ensuring correct interpretation and management.

There is variation in interpretation of AXR signs among clinicians ${ }^{13-15}$ and also among different professional groups. No formal training is currently offered to paediatricians.

We present a local guideline based on agreement among groups of professionals in order to standardise interpretation.

\section{CONCLUSIONS}

AXR remains the modality of choice for diagnosis and follow-up of NEC. Clinical correlation is important. The standardisation of X-ray interpretation aims to avoid cases of misclassified NEC and thereby reduce the number of babies subjected to periods of suspended oral feeding and unnecessary transfers, both of which have an overall negative impact on neurodevelopmental outcomes of preterm babies. Early identification of abnormal signs on X-rays will allow timely institution of medical therapy in cases of NEC.

\section{Search strategy}

Online OVID search was conducted using Embase from 1974 to 2018 and OVID Medline(R) from 1946 to 2018. The following were the keywords used: Necrotising enterocolitis OR Necrotizing Enterocolitis OR NEC AND X-ray OR imaging OR radiology OR radiograph AND Diagnosis OR signs. Eighty-four articles were obtained, of which 33 were found to be relevant. Referenced articles from some of the papers were also studied. A total of 19 articles were selected for final article submission.

\section{Clinical bottom lines}

- Although positive radiographic findings are of great predictive value, negative findings do not rule out the possibility of necrotising enterocolitis (NEC).

- Both pneumatosis and portal venous gas are pathognomonic signs of NEC; however, their absence or disappearance is not always a reassuring sign and therefore improvement should always be assessed clinically.

- Dilated bowel loops on X-ray are a non-specific sign and may also be present in normal premature babies; other clinical and pathophysiological signs are required to make a diagnosis of NEC.

- True 'bowel wall thickening' is rarely seen in NEC; the term 'separation of bowel loops' is more appropriate and is as such a non-specific sign.

- Abdominal USS is a useful adjunct to X-ray and can provide additional valuable information in patients with NEC.

\section{Test your knowledge}

Choose one answer for each of the following:

1. Which of the following signs is considered pathognomonic of necrotising enterocolitis (NEC)?
A. Pneumoperitoneum.
B. Pneumatosis intestinalis.
C. Distended bowel loops.
D. Flower pot sign.
E. Football sign.

2. Which sign is not suggestive of perforation?
A. Rigler sign.
B. Pneumoperitoneum.
C. Football sign.
D. Gas in patent processus vaginalis.
E. Intramural gas.

3. In a baby with NEC, which of the following may be considered an indication for surgical intervention?

A. Inverse flower pot sign.

B. Separation of bowel loops.

C. Presence of crescents in the right lower quadrant.

D. Rigler sign.

E. Soap bubbles in the right lower quadrant.

4. In figure 10, which of the following signs is not present?
A. Intramural gas.
B. Rigler sign.
C. Dilated bowel loops.
D. Air under the diaphragm.
E. Gas in the portal venous system.

5. What is the diagnosis in the infant based on the $\mathrm{X}$-ray findings in figure 10 ?
A. NEC.
B. NEC with perforation.
C. Volvulus.
D. Intussusception.
E. Focal intestinal perforation.

Answers to the quiz are at the end of the references. 
Collaborators Jessica Bolton, Simon Blackburn, Christina Kortsalioudaki, Bashar Al Deiri, Katie MacKinnon, Simona Rusu, Ekene Enunwa, Louiza Kontara, Ava Tzankova.

Contributors SR corresponded with the editor of the Interpretations section of $A D C$ Education \& Practice and wrote the several drafts of the article based on the guidance provided. She performed a literature search, regularly contacted and met all the coauthors for comments, drafted the final version of the article, and collated the images for submission. AK performed a literature search to support the drafting of the article in its initial stages. She participated in reviewing the future drafts of the article. $\mathrm{JIC}$ and $\mathrm{AH}-\mathrm{C}$ were instrumental in creating the 'proposed guide' within the article, on behalf of the GOSH surgical team and UCH Nutrition Group. They also both reviewed the many drafts of the article. JC provided the X-ray images for the article. PDH works closely with the neonatal and surgical teams at ULCH and GOSH. His input was crucial in the final review of the article.

Funding The authors have not declared a specific grant for this research from any funding agency in the public, commercial or not-for-profit sectors.

Competing interests None declared.

Patient consent Not required.

Provenance and peer review Commissioned; externally peer reviewed.

\section{REFERENCES}

1 Epelman M, Daneman A, Navarro OM, et al. Necrotizing enterocolitis: review of state-of-the-art imaging findings with pathologic correlation. Radiographics 2007;27:285-305.

2 Robinson JR, Rellinger EJ, Hatch LD, et al. Surgical necrotizing enterocolitis. Semin Perinatol 2017;41:70-9.

3 Lim JC, Golden JM, Ford HR. Pathogenesis of neonatal necrotizing enterocolitis. Pediatr Surg Int 2015;31:509-18.

4 Rees CM, Pierro A, Eaton S. Neurodevelopmental outcomes of neonates with medically and surgically treated necrotizing enterocolitis. Arch Dis Child Fetal Neonatal Ed 2007;92:F1938.

5 Hintz SR, Kendrick DE, Stoll BJ, et al. Neurodevelopmental and growth outcomes of extremely low birth weight infants after necrotizing enterocolitis. Pediatrics 2005;115:696-703.

6 Bell MJ, Ternberg JL, Feigin RD, et al. Neonatal necrotizing enterocolitis. Therapeutic decisions based upon clinical staging. Ann Surg 1978;187:1-7.

7 Kliegman RM, Walsh MC. Neonatal necrotizing enterocolitis: pathogenesis, classification, and spectrum of illness. Curr Probl Pediatr 1987;17:219-88.
8 Neu J, Walker WA. Necrotizing enterocolitis. N Engl J Med Overseas Ed 2011;364:255-64.

9 Buonomo C. The radiology of necrotizing enterocolitis. Radiol Clin North Am 1999;37:1187-98.

10 Tam AL, Camberos A, Applebaum H. Surgical decision making in necrotizing enterocolitis and focal intestinal perforation: Predictive value of radiologic findings. J Pediatr Surg 2002;37:1688-91.

11 Garbi-Goutel A, Brévaut-Malaty V, Panuel M, et al. Prognostic value of abdominal sonography in necrotizing enterocolitis of premature infants born before 33 weeks gestational age. $J$ Pediatr Surg 2014;49:508-13.

12 Silva CT, Daneman A, Navarro OM, et al. Correlation of sonographic findings and outcome in necrotizing enterocolitis. Pediatr Radiol 2007;37:274-82.

13 El-Kady S, Petel D, Baird R. Inter-rater agreement in the evaluation of abdominal radiographs for necrotizing enterocolitis. J Pediatr Surg 2014;49:733-5.

14 Rehan VK, Seshia MM, Johnston B, et al. Observer variability in interpretation of abdominal radiographs of infants with suspected necrotizing enterocolitis. Clin Pediatr 1999;38:637-43.

15 Di Napoli A, Di Lallo D, Perucci CA, et al. Inter-observer reliability of radiological signs of necrotising enterocolitis in a population of high-risk newborns. Paediatr Perinat Epidemiol 2004;18:80-7.

16 Williams H. Perforation: how to spot free intraperitoneal air on abdominal radiograph. Archives of Disease in Childhood Education and Practice 2006;91:ep54-ep57.

17 Rampton JW. The football sign. Radiology 2004;231:81-2.

18 Wexler HA. The persistent loop sign in neonatal necrotizing enterocolitis: a new indication for surgical intervention? Radiology 1978;126:201-4.

19 Leonard T, Johnson JF, Pettett PG. Critical evaluation of the persistent loop sign in necrotizing enterocolitis. Radiology 1982;142:385-6.

\section{Answers to the multiple choice questions}

1. B; 2. E; 3. D; 4. E; 5. B. 